\title{
HYDRODYNAMIC REDUCTIONS AND SOLUTIONS OF A UNIVERSAL HIERARCHY
}

\author{
L. Martínez Alonso ${ }^{1}$ and A. B. Shabat ${ }^{2}$ \\ ${ }^{1}$ Departamento de Física Teórica II, Universidad Complutense \\ E28040 Madrid, Spain \\ ${ }^{2}$ Landau Institute for Theoretical Physics \\ RAS, Moscow 117 334, Russia
}

\begin{abstract}
The diagonal hydrodynamic reductions of a hierarchy of integrable hydrodynamic chains are explicitly characterized. Their compatibility with previously introduced reductions of differential type is analyzed and their associated class of hodograph solutions is discussed.
\end{abstract}

Key words: Hydrodynamic Systems, Differential Reductions, Hodograph Solutions. MSC: 35L40,58B20. 


\section{Introduction}

In a series of papers [1-4] we have considered an infinite hierarchy of integrable systems [5] which admits many interesting $(1+1)$-dimensional reductions like the Burgers, KdV and NLS hierarchies as well as different classes of energy-dependent hierarchies [6]- 9] including the Camassa-Holm model also [10]. It motivated the term universal hierarchy we proposed in [1].

This universal hierarchy can be defined in terms of a generating function $G=G(\lambda, \boldsymbol{x})$ depending on an spectral parameter $\lambda$ and an infinite set of variables $\boldsymbol{x}:=\left(\ldots, x_{-1}, x_{0}, x_{1}, \ldots\right)$, which admits expansions

$$
\begin{aligned}
& G=1+\frac{g_{1}(\boldsymbol{x})}{\lambda}+\frac{g_{2}(\boldsymbol{x})}{\lambda^{2}}+\cdots, \quad \lambda \rightarrow \infty, \\
& G=b_{0}(\boldsymbol{x})+b_{1}(\boldsymbol{x}) \lambda+b_{2}(\boldsymbol{x}) \lambda^{2}+\cdots, \quad \lambda \rightarrow 0 .
\end{aligned}
$$

The hierarchy is provided by the system of flows

$$
\partial_{n} G=\left\langle A_{n}, G\right\rangle, \quad n \in \mathbb{Z},
$$

where

$$
\langle U, V\rangle:=U\left(\partial_{x} V\right)-\left(\partial_{x} U\right) V
$$

and

$$
\begin{aligned}
A_{n}: & =\lambda^{n}+g_{1}(\boldsymbol{x}) \lambda^{n-1}+\cdots+g_{n-1}(\boldsymbol{x}) \lambda+g_{n}(\boldsymbol{x}), \quad n \geq 0 \\
A_{-n}: & =\frac{b_{0}(\boldsymbol{x})}{\lambda^{n}}+\frac{b_{1}(\boldsymbol{x})}{\lambda^{n-1}}+\cdots+\frac{b_{n-1}(\boldsymbol{x})}{\lambda}, \quad n>0 .
\end{aligned}
$$

In terms of the coefficients $\left\{g_{n}(\boldsymbol{x})\right\}_{n \geq 1} \bigcup\left\{b_{n}(\boldsymbol{x})\right\}_{n \geq 0}$ the system (3) becomes a hierarchy of hydrodynamic chains.

An alternative and useful formulation of (3) is obtained by introducing the generating function

$$
H:=\frac{1}{G}
$$

Thus Eq.(3) is equivalent to

$$
\partial_{n} H=\partial_{x}\left(A_{n} H\right), \quad n \in \mathbb{Z},
$$


which means, in particular, that the coefficients $\left\{h_{n}(\boldsymbol{x})\right\}_{n \geq 1}$ supply an infinite set of conservation laws for (3).

The hierarchy (3) forms a compatible system. Indeed, as a consequence of (3) one derives the consistency conditions [1]- [4]

$$
\partial_{n} A_{m}-\partial_{m} A_{n}=\left\langle A_{n}, A_{m}\right\rangle, \quad n, m \in \mathbb{Z},
$$

It is important to notice that (8) implies that the pencil of differential forms

$$
\omega(\lambda):=\sum_{n \in \mathbb{Z}}\left(H A_{n}\right) \mathrm{d} x_{n}
$$

is closed with respect to the variables $\boldsymbol{x}$ since it satisfies

$$
\begin{gathered}
\partial_{m}\left(H A_{n}\right)-\partial_{n}\left(H A_{m}\right)=\partial_{m} H A_{n}-\partial_{n} H A_{m}+H\left(\partial_{m} A_{n}-\partial_{n} A_{m}\right) \\
=\partial_{x}\left(H A_{m}\right) A_{n}-\partial_{x}\left(H A_{n}\right) A_{m}+H\left\langle A_{n}, A_{m}\right\rangle=0 .
\end{gathered}
$$

The potential function $Q=Q(\lambda, \boldsymbol{x})$ corresponding to $\omega$

$$
\mathrm{d} Q=\omega
$$

leads to another useful formulation of our hierarchy. Indeed, according to (9)

$$
\partial_{n} Q=A_{n} \partial_{x} Q, \quad n \in \mathbb{Z} .
$$

Moreover, (11) is completely determined by $Q$ as

$$
G=\frac{1}{\partial_{x} Q}
$$

In Section 2 of this paper the formulation (111) is applied to prove that (3) includes multidimensional models such as

$$
\begin{aligned}
& u_{t x}=u_{x} u_{y z}-u_{y x} u_{z}, \\
& u_{y y}=u_{y} u_{x z}-u_{x y} u_{z}, \\
& u_{z x}=u_{x} u_{y y}-u_{x y} u_{y}, \\
& \left(\frac{u_{t}}{u_{x}}\right)_{t}=\left(\frac{u_{y}}{u_{x}}\right)_{z}, \\
& \left(\frac{u_{t}}{u_{x}}\right)_{t}=\left(\frac{u_{y}}{u_{x}}\right)_{x} .
\end{aligned}
$$


Section 3 deals with the main aim of the present paper: to characterize the hydrodynamic reductions of (3). These reductions are given by the solutions of (13) of the form $G=G(\lambda, \boldsymbol{R})$, where $\boldsymbol{R}=\left(R^{1}, \ldots, R^{N}\right)$ denotes a finite set of functions (Riemann invariants) satisfying a system of hydrodynamic equations of diagonal form

$$
\partial_{n} R^{i}=\Lambda_{n}^{i}(\boldsymbol{R}) \partial_{x} R^{i}, \quad n \in \mathbb{Z}
$$

This type of reductions appeared in the context of the dispersionless KP hierarchy [11]- 14] and has been also used in 15. to characterize the integrability of $(2+1)$-dimensional quasilinear systems. In the present paper we study these reductions for the whole set of flows of the hierarchy(3) and we obtain their explicit form . Our results are summarized in Theorem 1. It should be noticed that a similar analysis for the first member $\left(t_{1}\right.$-flow) of (31) has been recently performed in [16]. The compatibility between hydrodynamic and differential reductions considered in part 2 of Section 3. The paper finishes with a discussion of the solutions supplied by the generalized hodograph method [17.

The following notation conventions are henceforth used. Firstly, $G_{\infty}$ and $G_{0}$ stand for the expansions (11) (2) of $G$ as $\lambda \rightarrow \infty$ and $\lambda \rightarrow 0$, respectively. Furthermore, let $\mathbb{V}$ be the space of formal Laurent series

$$
V=\sum_{n=-\infty}^{\infty} a_{n} \lambda^{n} .
$$

We will denote by $\mathbb{V}_{r, s}(r \leq s)$ the subspaces of elements

$$
V=\sum_{n=r}^{s} a_{n} \lambda^{n} .
$$

and by $P_{r, s}: \mathbb{V} \mapsto \mathbb{V}_{r, s}$ the corresponding projectors. Given $V \in \mathbb{V}$ we will also denote

$$
(V)_{r, s}:=P_{r, s}(V) .
$$

In particular, notice that we can write

$$
A_{n}=\left(\lambda^{n} G_{\infty}\right)_{0,+\infty}, \quad A_{-n}=\left(\lambda^{-n} G_{0}\right)_{-\infty,-1}, \quad n \geq 1
$$




\section{Integrable models arising in the hierarchy}

\subsection{Multidimensional models}

If we use the potential function $Q(\lambda, \boldsymbol{x})$ for the differential form (9)

$$
\mathrm{d} Q=\sum_{n \in \mathbb{Z}}\left(H A_{n}\right) \mathrm{d} x_{n},
$$

then from (11) one readily deduces that $Q$ admits expansions of the form

$$
\begin{aligned}
& Q=\sum_{n \geq 0} \lambda^{n} x_{n}+\frac{q_{1}(\boldsymbol{x})}{\lambda}+\frac{q_{2}(\boldsymbol{x})}{\lambda^{2}}+\cdots, \quad \lambda \rightarrow \infty, \\
& Q=\sum_{n \geq 1} \frac{x_{n}}{\lambda^{n}}+p_{0}(\boldsymbol{x})+p_{1}(\boldsymbol{x}) \lambda+p_{2}(\boldsymbol{x}) \lambda^{2}+\cdots, \quad \lambda \rightarrow 0 .
\end{aligned}
$$

By substituting (20) into (19) and by identifying coefficients of equal powers of $\lambda$ one obtains formulas for the differentials of the functions $q_{n}(\boldsymbol{x})$

and $p_{n}(\boldsymbol{x})$ in terms of the coefficients of the expansions (11) and (2). For example, the simplest ones are

$$
\begin{aligned}
& \mathrm{d} q_{1}=b_{0} \mathrm{~d} x_{-1}+\sum_{n \geq 1}\left(b_{n} \mathrm{~d} x_{-n-1}-g_{n} \mathrm{~d} x_{n-1}\right), \\
& \mathrm{d} p_{0}=\frac{1}{b_{0}}\left(\mathrm{~d} x_{0}+\sum_{n \geq 1}\left(g_{n} \mathrm{~d} x_{n}-b_{n} \mathrm{~d} x_{-n}\right)\right) .
\end{aligned}
$$

They imply

$$
\begin{aligned}
\mathrm{d} q_{1} & =\frac{1}{\partial_{x} p_{0}}\left(\mathrm{~d} x_{-1}-\sum_{n \neq-1} \partial_{n+1} p_{0} \mathrm{~d} x_{n}\right) \\
\mathrm{d} p_{0} & =\frac{1}{\partial_{-1} q_{1}}\left(\mathrm{~d} x_{0}-\sum_{n \neq 0} \partial_{n-1} q_{1} \mathrm{~d} x_{n}\right) .
\end{aligned}
$$

Permutability of crossing derivatives of $q_{1}$ and $p_{0}$ in these identities lead at once to multidimensional nonlinear equations for the functions $g_{n}$ and $b_{n}$. For example, starting from

$$
\partial_{m} \partial_{-1} q_{1}=\partial_{-1} \partial_{m} q_{1}, \quad m \neq-1
$$


and using (24), the following nonlinear equation results

$$
\partial_{n} \partial_{0} p_{0}=\partial_{x} p_{0}\left(\partial_{-1} \partial_{n+1} p_{0}\right)-\left(\partial_{-1} \partial_{x} p_{0}\right) D_{n+1} p_{0}, \quad n \neq-1 .
$$

In the same way, from the crossing relation

$$
\partial_{m} \partial_{n} q_{1}=\partial_{n} \partial_{m} q_{1}, \quad m, n \neq-1,
$$

and (24) we get the following nonlinear equation

$$
\partial_{m}\left(\frac{\partial_{n+1} p_{0}}{\partial_{x} p_{0}}\right)=\partial_{n}\left(\frac{\partial_{m+1} p_{0}}{\partial_{x} p_{0}}\right), \quad m, n \neq-1 .
$$

The same type of equations can be derived for $q_{1}$. The different choices available for $n, m$ in the equations (26) and (27) give rise to the models (13)-(17).

\subsection{2-dimensional integrable models}

In [1-3] we developed a theory of differential reductions of our hierarchy based on imposing differential constraints on $G \approx G_{\infty}$ of the form

$$
\left(\mathcal{F}\left(\lambda, G, G_{x}, G_{x x}, \ldots\right)\right)_{-\infty,-1}=0, \quad x:=x_{0} .
$$

In particular the following three classes of reductions associated to arbitrary polynomials $a=a(\lambda)$ in $\lambda$ were characterized:

\section{Zero-order reductions}

$$
a(\lambda) G=U(\lambda, \boldsymbol{x}), \quad U:=(a(\lambda) G)_{0,+\infty},
$$

First-order reductions

$$
G_{x}+a(\lambda)=U(\lambda, \boldsymbol{x}) G, \quad U:=\left(\frac{a}{G}\right)_{0,+\infty},
$$




\section{Second-order reductions}

$$
\frac{1}{2} G G_{x x}-\frac{1}{4} G_{x}^{2}+a(\lambda)=U(\lambda, \boldsymbol{x}) G^{2}, \quad U:=\left(\frac{a}{G^{2}}\right)_{0,+\infty} .
$$

The first-order reduction for a linear function $a(\lambda)$ determines the Burgers hierarchy. On the other hand, under the differential constraints (31) the hierarchy (18) describes the KdV hierarchy and its generalizations associated to energy-dependent Schrödinger spectral problems. In particular, the linear and quadratic choices for $a(\lambda)$ lead to the KdV (Korteweg-deVries) and NLS (Nonlinear-Schrödinger) hierarchies, respectively. Indeed, if we define the functions $\psi(\lambda, \boldsymbol{x})$ by

$$
\psi(\lambda, \boldsymbol{x}):=\exp \left(D_{x}^{-1} \phi\right), \quad \phi:=-\frac{1}{2} \frac{H_{x}}{H} \pm \sqrt{a(\lambda)} H
$$

then from (31) it is straightforward to deduce that

$$
\begin{aligned}
\partial_{n} \psi & =-\frac{1}{2}\left(\partial_{n} \log H\right) \psi \pm \sqrt{a} A_{n} H \psi \\
& =A_{n}\left(-\frac{1}{2} D_{x} \log H \pm \sqrt{a} H\right) \psi-\frac{1}{2} A_{n, x} \psi \\
& =A_{n} \psi_{x}-\frac{1}{2} A_{n, x} \psi \\
\psi_{x x} & =\left(\phi_{x}+\phi^{2}\right) \psi=\left(\left\{D_{x}, H\right\}+a H^{2}\right) \psi=U \psi
\end{aligned}
$$

In other words, the functions $\psi$ are wave functions for the integrable hierarchies associated to energy-dependent Schrödinger problems. The evolution law of the potential function $U$ under the flows (18) can be determined from the equation

$$
\partial_{n} U=-\frac{1}{2} A_{n, x x x}+2 U A_{n, x}+U_{x} A_{n}
$$

which arises as an straightforward consequence of (18) and (31).

Additional reduced hierarchies including nonlinear integrable models such as the Camassa-Holm equation can be also deduced (see [4]). 


\section{Hydrodynamic reductions and solutions}

\subsection{Hydrodynamic reductions}

Let us consider now the hydrodynamic reductions of (3). We look for classes a solutions

$$
G=G(\lambda, \boldsymbol{R}),
$$

of (3) where $\boldsymbol{R}=\left(R^{1}, \ldots, R^{N}\right)$ satisfies a infinite system of hydrodynamic equations of diagonal form (18). Our aim is to characterize both the form of $G=G(\lambda, \boldsymbol{R})$ and the characteristic speeds $\Lambda_{n}^{i}$ defining the system (18). By substituting (35) into (3) then by using (18) the identification of coefficients of the derivatives $\partial_{x} R^{i}, i=1, \ldots, N$ implies

$$
\left(D_{i} G\right) \Lambda_{n}^{i}=A_{n}\left(D_{i} G\right)-\left(D_{i} A_{n}\right) G, \quad 1 \leq i \leq N, \quad n \in \mathbb{Z}
$$

where

$$
D_{i}:=\frac{\partial}{\partial R^{i}}
$$

In addition to these equations we impose the requirement of the commutativity of the flows (18), which is equivalent to the following restrictions on the characteristic speeds

$$
\frac{D_{j} \Lambda_{n}^{i}}{\Lambda_{n}^{j}-\Lambda_{n}^{i}}=\frac{D_{j} \Lambda_{m}^{i}}{\Lambda_{m}^{j}-\Lambda_{m}^{i}}, \quad i \neq j, \quad m \neq n .
$$

We start our analysis by considering the positive flows $n \geq 1$. From (35) we get the following system for $G \approx G_{\infty}$

$$
\left(D_{i} G\right) \Lambda_{n}^{i}=\left(\lambda^{n} G\right)_{-\infty, 0}\left(D_{i} G\right)-\left(D_{i}\left(\lambda^{n} G\right)_{-\infty, 0}\right) G, \quad n \geq 1 .
$$

By substituting in these equations the expansion $G \approx G_{\infty}$ and identifying the coefficients in $\frac{1}{\lambda}$ and $\frac{1}{\lambda^{2}}$ we get

$$
\begin{aligned}
& D_{i} g_{n+1}=\Lambda_{n}^{i} D_{i} g_{1}, \\
& D_{i} g_{n+2}=\Lambda_{n}^{i} D_{i} g_{2}+g_{n+1}\left(D_{i} g_{1}\right)-\left(D_{i} g_{n+1}\right) g_{1} .
\end{aligned}
$$

As an immediate consequence it follows

$$
\Lambda_{n+1}^{i}=g_{n+1}+\Lambda_{n}^{i}\left(\Lambda_{1}^{i}-g_{1}\right)
$$


which implies

$$
\Lambda_{n}^{i}=A_{n}\left(\lambda=\Lambda_{1}^{i}-g_{1}\right), \quad n \geq 1 .
$$

We now look for a system characterizing $\Lambda_{1}^{i}$ and $g_{1}$. To this end we differentiate (38) and find

$$
\begin{aligned}
& D_{j} D_{i} g_{n+1}=D_{j} D_{i} g_{1} \Lambda_{n}^{i}+D_{i} g_{1} D_{j} \Lambda_{n}^{i} \\
& =D_{i} D_{j} g_{1} \Lambda_{n}^{j}+D_{j} g_{1} D_{i} \Lambda_{n}^{i}
\end{aligned}
$$

so that

$$
D_{i j} g_{1}=\frac{D_{j} \Lambda_{n}^{i}}{\Lambda_{n}^{j}-\Lambda_{n}^{i}} D_{i} g_{1}+\frac{D_{i} \Lambda_{n}^{j}}{\Lambda_{n}^{i}-\Lambda_{n}^{j}} D_{j} g_{1},
$$

and from (36) we may write

$$
D_{i j} g_{1}=\frac{D_{j} \Lambda_{1}^{i}}{\Lambda_{1}^{j}-\Lambda_{1}^{i}} D_{i} g_{1}+\frac{D_{i} \Lambda_{1}^{j}}{\Lambda_{1}^{i}-\Lambda_{1}^{j}} D_{j} g_{1}, \quad i \neq j .
$$

On the other hand according to (40)

$$
\Lambda_{2}^{i}=g_{2}+\Lambda_{1}^{i}\left(\Lambda_{1}^{i}-g_{1}\right),
$$

so that from (36) and with the help of (38) we find

$$
\frac{D_{j} \Lambda_{1}^{i}}{\Lambda_{1}^{j}-\Lambda_{1}^{i}}=\frac{D_{j} \Lambda_{2}^{i}}{\Lambda_{2}^{j}-\Lambda_{2}^{i}}=\frac{D_{j} \Lambda_{1}^{i}\left(2 \Lambda_{1}^{i}-g_{1}\right)+\left(\Lambda_{1}^{j}-\Lambda_{1}^{i}\right) D_{j} g_{1}}{\left(\Lambda_{1}^{j}-\Lambda_{1}^{i}\right)\left(\Lambda_{1}^{j}+\Lambda_{1}^{i}-g_{1}\right)}, \quad i \neq j
$$

which reduces to

$$
D_{j} \Lambda_{1}^{i}=D_{j} g_{1}, \quad i \neq j
$$

In this way

$$
\Lambda_{1}^{i}=g_{1}+f^{i}\left(R^{i}\right)
$$

where the functions $f^{i}$ are arbitrary. By using this result in (42) it follows

$$
D_{i j} g_{1}=0, \quad i \neq j
$$

so that

$$
g_{1}=\sum_{k=1}^{N} h^{k}\left(R^{k}\right)
$$

where the functions $h^{k}$ are arbitrary. 
By using these results we may determine $G(\lambda, \boldsymbol{R})$ since from the equation (35) with $n=1$

$$
D_{i} \ln G=\frac{D_{i} A_{1}}{A_{1}-\Lambda_{1}^{i}}=\frac{\dot{h}^{i}\left(R^{i}\right)}{\lambda-f^{i}\left(R^{i}\right)},
$$

where $\dot{h}^{i}:=D_{i} h^{i}$. Thus we get

$$
G(\lambda, \boldsymbol{R})=\exp \left(\sum_{i=1}^{N} \int^{R^{i}} \frac{\dot{h}^{i}\left(R^{i}\right)}{\lambda-f^{i}\left(R^{i}\right)} \mathrm{d} R^{i}\right),
$$

where the undefinite integrations are determined up to a function of $\lambda$ decaying at $\lambda \rightarrow \infty$. The expression (45) coincides with the generating function of the conservation laws densities found in [16].

Let us now determine the characteristic speeds $\Lambda_{n}^{i}$ for the negative flows $n \leq-1$. The equations (35) imply the following system for $G \approx G_{0}$

$$
\left(D_{i} G\right) \Lambda_{-n}^{i}=\left(\lambda^{-n} G\right)_{0, \infty}\left(D_{i} G\right)-\left(D_{i}\left(\lambda^{-n} G\right)_{0, \infty}\right) G, \quad n \geq 1 .
$$

Then by inserting the expansion $G \approx G_{0}$ of (2) and identifying the coefficients in $\lambda^{0}$ and $\lambda$ we get

$$
\begin{aligned}
& D_{i} b_{n}=\left(b_{n}+\Lambda_{-n}^{i}\right) D_{i} \ln b_{0}, \\
& b_{0} D_{i} b_{n+1}=\left(b_{n}+\Lambda_{n}^{i}\right) D_{i} b_{1}+b_{n+1}\left(D_{i} b_{0}\right)-\left(D_{i} b_{n}\right) b_{1} .
\end{aligned}
$$

It implies the following recurrence relation for the characteristic speeds

$$
\Lambda_{-n-1}^{i}=\frac{\Lambda_{-1}^{i}}{b_{0}}\left(b_{n}+\Lambda_{-n}^{i}\right)
$$

which implies

$$
\Lambda_{-n}^{i}=A_{-n}\left(\lambda=\frac{b_{0}}{\Lambda_{-1}^{i}}\right), \quad n \geq 1 .
$$

The only unknown now is $\Lambda_{-1}^{i}$ since according to (45)

$$
b_{0}=\exp \left(-\sum_{i=1}^{N} \int^{R^{i}} \frac{\dot{h}^{i}\left(R^{i}\right)}{f^{i}\left(R^{i}\right)} \mathrm{d} R^{i}\right) .
$$

To find $\Lambda_{-1}^{i}$ we use (49) for $n=1$

$$
\Lambda_{-2}^{i}=\frac{\Lambda_{-1}^{i}}{b_{0}}\left(b_{1}+\Lambda_{-1}^{i}\right)
$$


and the commutativity condition for the $n=-1$ and $n=-2$ flows

$$
\frac{D_{j} \Lambda_{-1}^{i}}{\Lambda_{-1}^{j}-\Lambda_{-1}^{i}}=\frac{D_{j} \Lambda_{-2}^{i}}{\Lambda_{-2}^{j}-\Lambda_{-2}^{i}} .
$$

Thus by eliminating $\lambda_{-2}^{i}$ one finds at once

$$
D_{j} \ln \Lambda_{-1}^{i}=D_{j} \ln b_{0}, \quad i \neq j .
$$

Therefore,

$$
\Lambda_{-1}^{i}=b_{0} g^{i}\left(R^{i}\right)
$$

with $g^{i}$ being arbitrary functions.

At this point we have determined all the unknowns of our problem. However, in our calculation we only used a subset of the equations required, so we must prove that our solution satisfies the full system of equations (35)-(36).

Let us begin with (35) for $n \geq 1$

$$
D_{i} \ln G=\frac{D_{i} A_{n}}{A_{n}-\Lambda_{n}^{i}}, \quad n \geq 1,
$$

which according to (41), (43)-(45) reads

$$
D_{i} A_{n}(\lambda)=\frac{\dot{h}^{i}}{\lambda-f^{i}}\left(A_{n}(\lambda)-A_{n}\left(\lambda=f^{i}\right)\right) .
$$

To prove this identity we express $A_{n}(\lambda)$ as

$$
\begin{aligned}
A_{n}(\lambda) & =\left(\lambda^{n} G\right)_{0, \infty}=\frac{1}{2 \pi i} \int_{\gamma_{\infty}} \frac{\left(\widetilde{\lambda}^{n} G(\widetilde{\lambda})\right)_{0, \infty}}{\widetilde{\lambda}-\lambda} \mathrm{d} \widetilde{\lambda}=\frac{1}{2 \pi i} \int_{\gamma_{\infty}} \frac{\widetilde{\lambda}^{n} G(\widetilde{\lambda})}{\widetilde{\lambda}-\lambda} \mathrm{d} \widetilde{\lambda} \\
& =\frac{1}{2 \pi i} \int_{\gamma_{\infty}} \frac{\widetilde{\lambda}^{n}}{\widetilde{\lambda}-\lambda} \exp \left(\sum_{i=1}^{N} \int^{R^{i}} \frac{\dot{h}^{i}}{\widetilde{\lambda}-f^{i}} \mathrm{~d} R^{i}\right) \mathrm{d} \widetilde{\lambda},
\end{aligned}
$$

where $\gamma_{\infty}$ is a positively oriented closed loop around $\infty$ in the complex plane of $\widetilde{\lambda}$ ( $\lambda$ and $f^{i}$ are assumed to lie inside the loop). Now by differentiating this expression with respect to $R^{i}$ one finds

$$
\begin{aligned}
D_{i} A_{n}(\lambda) & =\frac{1}{2 \pi i} \int_{\gamma_{\infty}} \frac{\dot{h}^{i} \widetilde{\lambda}^{n} G(\widetilde{\lambda})}{(\widetilde{\lambda}-\lambda)\left(\widetilde{\lambda}-f^{i}\right)} \mathrm{d} \widetilde{\lambda}=\frac{1}{2 \pi i} \int_{\gamma_{\infty}} \frac{\dot{h}^{i} A_{n}(\widetilde{\lambda})}{(\widetilde{\lambda}-\lambda)\left(\widetilde{\lambda}-f^{i}\right)} \mathrm{d} \widetilde{\lambda} \\
& =\frac{\dot{h}^{i}}{\lambda-f^{i}}\left(A_{n}(\lambda)-A_{n}\left(\lambda=f^{i}\right)\right),
\end{aligned}
$$


which proves (52). This identity leads also at once to (36) since it implies

$$
\frac{D_{j} A_{n}\left(\lambda=f^{i}\right)}{A_{n}\left(\lambda=f^{j}\right)-A_{n}\left(\lambda=f^{i}\right)}=\frac{\dot{h}^{j}}{f^{j}-f^{i}}, \quad i \neq j,
$$

which means that

$$
\frac{D_{j} \Lambda_{n}^{i}}{\Lambda_{n}^{j}-\Lambda_{n}^{i}}=\frac{D_{j} \Lambda_{1}^{i}}{\Lambda_{1}^{j}-\Lambda_{1}^{i}}, \quad n>1 .
$$

Let us consider now (35) for $n \leq-1$

$$
D_{i} \ln G=\frac{D_{i} A_{-n}}{A_{-n}-\Lambda_{-n}^{i}}, \quad n \geq 1 .
$$

From (45), (150) and (51) it takes the form

$$
D_{i} A_{-n}(\lambda)=\frac{\dot{h}^{i}}{\lambda-f^{i}}\left(A_{-n}(\lambda)-A_{-n}\left(\lambda=\frac{1}{g^{i}}\right)\right) .
$$

In order to proof (53) we express $A_{-n}(\lambda)$ in the form

$$
\begin{aligned}
A_{-n}(\lambda) & =\left(\lambda^{-n} G\right)_{-\infty,-1}=\frac{1}{2 \pi i} \int_{\gamma_{0}} \frac{\left(\widetilde{\lambda}^{-n} G(\widetilde{\lambda})\right)_{-\infty,-1}}{\widetilde{\lambda}-\lambda} \mathrm{d} \widetilde{\lambda}=\frac{1}{2 \pi i} \int_{\gamma_{0}} \frac{\tilde{\lambda}^{-n} G(\widetilde{\lambda})}{\widetilde{\lambda}-\lambda} \mathrm{d} \tilde{\lambda} \\
& =\frac{1}{2 \pi i} \int_{\gamma_{0}} \frac{\widetilde{\lambda}^{-n}}{\widetilde{\lambda}-\lambda} \exp \left(\sum_{i=1}^{N} \int^{R^{i}} \frac{\dot{h}^{i}}{\widetilde{\lambda}-f^{i}} \mathrm{~d} R^{i}\right) \mathrm{d} \tilde{\lambda},
\end{aligned}
$$

where $\gamma_{0}$ is a closed small loop with negative orientation around $\widetilde{\lambda}=0$ ( $\lambda$ and $f^{i}$ are assumed to lie outside the loop ). By differentiating with respect to $R^{i}$ it yields

$$
\begin{aligned}
D_{i} A_{-n}(\lambda) & =\frac{1}{2 \pi i} \int_{\gamma_{0}} \frac{\dot{h}^{i} \widetilde{\lambda}^{-n} G(\widetilde{\lambda})}{(\widetilde{\lambda}-\lambda)\left(\widetilde{\lambda}-f^{i}\right)} \mathrm{d} \widetilde{\lambda} \\
& =\frac{1}{2 \pi i} \int_{\gamma_{0}} \frac{\dot{h}^{i} A_{-n}(\widetilde{\lambda})}{(\widetilde{\lambda}-\lambda)\left(\widetilde{\lambda}-f^{i}\right)} \mathrm{d} \widetilde{\lambda}=\frac{\dot{h}^{i}}{\lambda-f^{i}}\left(A_{-n}(\lambda)-A_{-n}\left(\lambda=f^{i}\right)\right),
\end{aligned}
$$

Hence (53) holds if we set

$$
g^{i}\left(R^{i}\right)=\frac{1}{f^{i}\left(R^{i}\right)}
$$


The remaining commutativity conditions (36) follow at once.

Therefore we may summarize our analysis in the next theorem

Theorem 1. The hydrodynamic reductions of the hierarchy (31) are determined by

$$
\begin{aligned}
& G(\lambda, \boldsymbol{R})=\exp \left(\sum_{i=1}^{N} \int R^{i} \frac{\dot{h}^{i}\left(R^{i}\right)}{\lambda-f^{i}\left(R^{i}\right)} \mathrm{d} R^{i}\right), \\
& \partial_{n} R^{i}=\Lambda_{n}^{i}(\boldsymbol{R}) \partial_{x} R^{i}, \quad \Lambda_{n}^{i}(\boldsymbol{R}):=A_{n}\left(\lambda=f^{i}\left(R^{i}\right)\right),
\end{aligned}
$$

where the functions $h^{i}$ and $f^{i}$ are arbitrary.

Since the systems (18) are invariant under local transformations of the form $R^{i} \rightarrow \widetilde{R^{i}}\left(R^{i}\right)$, without loss of generality we will henceforth set

$$
h^{i}\left(R^{i}\right)=R^{i}
$$

so that the form of the reduced generating function is

$$
G(\lambda, \boldsymbol{R})=\exp \left(\sum_{i=1}^{N} \int^{R^{i}} \frac{\mathrm{d} R^{i}}{\lambda-f^{i}\left(R^{i}\right)}\right)
$$

In this way some of the simplest hydrodynamic reductions (54) are given by

$$
\begin{aligned}
& f^{i}\left(R^{i}\right)=c_{i}, \quad G=\exp \left(\sum_{i=1}^{N} \frac{R^{i}}{\lambda-c_{i}}\right) \\
& f^{i}\left(R^{i}\right)=-\frac{R^{i}}{\epsilon_{i}}, \quad G=\prod_{i=1}^{N}\left(\frac{\epsilon_{i} \lambda+R^{i}}{\epsilon_{i} \lambda+\lambda_{i}}\right)^{\epsilon_{i}} .
\end{aligned}
$$

We also notice that the characteristic velocities of the hydrodynamic systems (55) can be written in terms of the Schur polynomials

$$
\exp \left(\sum_{n \geq 1} k^{n} x_{n}\right)=\sum_{n \geq 0} k^{n} S_{n}\left(x_{1}, \ldots, x_{n}\right),
$$


as

$$
\begin{aligned}
& \Lambda_{n}^{i}(\boldsymbol{R})=\sum_{j=0}^{n} S_{j}\left(I_{1}, \ldots, I_{j}\right)\left(f^{i}\left(R^{i}\right)\right)^{n-j}, \quad n \geq 0, \\
& \Lambda_{-n}^{i}(\boldsymbol{R})=\exp \left(-I_{0}\right) \sum_{j=0}^{n-1} S_{j}\left(I_{-1}, \ldots, I_{-j}\right)\left(f^{i}\left(R^{i}\right)\right)^{-n+j}, \quad n>0,
\end{aligned}
$$

where

$$
I_{n}:=\operatorname{sgn}(n) \sum_{i=1}^{N} \int^{R^{i}}\left(f^{i}\left(R^{i}\right)\right)^{n-1} \mathrm{~d} R^{i}
$$

\subsection{Compatibility with differential reductions}

A natural question is to find the hydrodynamic reductions compatible with the differential reductions (30) and (31). Let us prove the following result:

Theorem 2. The only hydrodynamic reductions (57) compatible with either first or second order differential constraints are characterized by

$$
f^{i}\left(R^{i}\right)=-R^{i}+c_{i}, \quad i=1, \ldots, N,
$$

which correspond to generating functions of the form

$$
G(\lambda, \boldsymbol{R})=\alpha(\lambda) \prod_{i=1}^{N}\left(\lambda+R^{i}-c_{i}\right)
$$

Proof. If we substitute (57) into the differential constraint (31) for secondorder differential reductions we get

$$
\frac{a(\lambda)}{G^{2}}=U-\frac{1}{2} \sum_{i}\left(\dot{f}^{i} \frac{\left(\partial_{x} R^{i}\right)^{2}}{\left(\lambda-f^{i}\right)^{2}}+\frac{\partial_{x x} R^{i}}{\lambda-f^{i}}\right)-\frac{1}{4}\left(\sum_{i} \frac{\partial_{x} R^{i}}{\lambda-f^{i}}\right)^{2} .
$$

This means that $G$ has a simple zero at each $\lambda=f^{i}\left(R^{i}\right)$ so that from (57) we have

$$
\exp \left(\int^{R^{i}} \frac{\mathrm{d} R^{i}}{\lambda-f^{i}\left(R^{i}\right)}\right)=\left(\lambda-f^{i}\left(R^{i}\right)\right) H\left(\lambda, R^{i}\right),
$$

where $H$ is different from zero at $\lambda=f^{i}\left(R^{i}\right)$. If we now differentiate with respect to $R^{i}$ we deduce that

$$
\frac{1}{\lambda-f^{i}}=-\frac{\dot{f}^{i}}{\lambda-f^{i}}+\mathcal{O}(1), \quad \lambda \rightarrow \infty .
$$


Therefore the statement of the theorem for second-order differential constraints follows. The corresponding proof for first-order constraints is similar.

\subsection{Hodograph solutions}

The general solution of the infinite system (55) is provided by the implicit generalized hodograph formula [17]

$$
x+\sum_{n \in \mathbb{Z}-\{0\}} \Lambda_{n}^{i}(\boldsymbol{R}) x_{n}=\Gamma^{i}(\boldsymbol{R}), \quad i=1, \ldots, N,
$$

where the functions $\Gamma^{i}$ are the general solution of the linear system

$$
\frac{D_{j} \Gamma^{i}}{\Gamma^{j}-\Gamma^{i}}=\frac{1}{f^{j}-f^{i}}, \quad i \neq j
$$

By introducing the potential function $\Phi(\boldsymbol{R})$

$$
\Gamma^{i}=D_{i} \Phi, \quad i=1, \ldots, N
$$

the system (61) reduces to the Laplace type form

$$
\left(f^{i}-f^{j}\right) D_{i} D_{j} \Phi=D_{i} \Phi-D_{j} \Phi, \quad i \neq j,
$$

the general solution of which depends on $N$ arbitrary functions of one variable.

In particular it is immediate to deduce [16] that the generating function (57) provides a one-parameter family of solutions of (62). Thus we can produce important solutions of (61) from linear superpositions of $G(\lambda, \boldsymbol{R})$. For example

$$
\begin{aligned}
& \Lambda_{n}^{i}=D_{i} \Phi, \quad \Phi:=\frac{1}{2 \pi i} \int_{\gamma_{\infty}} \lambda^{n} G(\lambda, \boldsymbol{R}) \mathrm{d} \lambda, \quad n \geq 0, \\
& \Lambda_{-n}^{i}=D_{i} \Phi, \quad \Phi:=\frac{1}{2 \pi i} \int_{\gamma_{0}} \lambda^{-n} G(\lambda, \boldsymbol{R}) \mathrm{d} \lambda, \quad n>0 .
\end{aligned}
$$

Furthermore, in several important cases the general solution of (62) can be written in terms of $G(\lambda, \boldsymbol{R})$.

\section{Examples}


If we set

$$
f^{i}\left(R^{i}\right)=\frac{R^{i}}{n}, \quad n=1,2, \ldots,
$$

then the generating function (non normalized as $\lambda \rightarrow \infty$ ) is

$$
G(\lambda, \boldsymbol{R})=\prod_{i=1}^{N}\left(\lambda-\frac{R^{i}}{n}\right)^{-n}
$$

Hence, by taking for each $i=1, \ldots, N$ a closed loop $\gamma_{i}$ in the complex $\lambda$ plane with positive orientation around $\lambda_{i}=\frac{R^{i}}{n}$, the general solution of (62) can be expressed as

$$
\Phi:=\frac{1}{2 \pi i} \sum_{i=1}^{N} \int_{\gamma_{i}} \phi_{i}(\lambda) G(\lambda, \boldsymbol{R}) \mathrm{d} \lambda
$$

where the functions $\phi_{i}(\lambda)$ are arbitrary. For example, if $n=1$ it takes the form

$$
\Phi=\sum_{i=1}^{N} \phi_{i}\left(R^{i}\right) \prod_{k \neq i} \frac{1}{R^{i}-R^{k}} .
$$

Similarly we may deal with the case

$$
f^{i}\left(R^{i}\right)=-\frac{R^{i}}{n}, \quad n=1,2, \ldots
$$

which leads to

$$
G(\lambda, \boldsymbol{R})=\prod_{i=1}^{N}\left(\lambda+\frac{R^{i}}{n}\right)^{n} .
$$

Now to generate the general solution of (62) we take for each $i=1, \ldots, N$ a path $\gamma_{i}\left(R^{i}\right)$ in the complex $\lambda$-plane ending at $\lambda_{i}=-R^{i} / n$ so that we can write

$$
\Phi:=\frac{1}{2 \pi i} \sum_{i=1}^{N} \int_{\gamma_{i}\left(R^{i}\right)} \phi_{i}(\lambda) G(\lambda, \boldsymbol{R}) \mathrm{d} \lambda,
$$

where the functions $\phi_{i}(\lambda)$ are arbitrary.

Let us consider in detail the case

$$
f^{i}\left(R^{i}\right)=-R^{i}, \quad i=1,2 .
$$


One finds

$$
\Phi=\left(\theta_{1}\left(-R^{1}\right)-\theta_{2}\left(-R^{2}\right)\right)\left(R^{1}-R^{2}\right)+2 \int^{-R^{1}} \theta_{1}(\lambda) \mathrm{d} \lambda+2 \int^{-R^{2}} \theta_{2}(\lambda) \mathrm{d} \lambda,
$$

with $\theta_{i}(\lambda)$ being arbitrary functions. A normalized generating function is given by

$$
G(\lambda, \boldsymbol{R})=\frac{\left(\lambda+R^{1}\right)\left(\lambda+R^{2}\right)}{\left(\lambda+\lambda_{0}\right)^{2}}, \quad \lambda_{0} \neq 0 .
$$

We may characterize the general solution of the hydrodynamic flows (55) corresponding to $n=-1$ and $n=-2$ by means of the hodograph formula

$$
x+A_{-1}\left(\lambda=-R^{i}\right) y+A_{-2}\left(\lambda=-R^{i}\right) z=D_{i} \Phi, \quad i=1,2,
$$

where $y:=x_{-1}, z:=x_{-2}$. From (63) one calculates

$$
b_{0}=c^{2} R^{1} R^{2}, \quad b_{1}=c^{2}\left(R^{1}+R^{2}\right)-2 c^{3} R^{1} R^{2}, \quad c:=\frac{1}{\lambda_{0}},
$$

and gets that the system (64) reads

$$
\begin{aligned}
& \left(x-c^{2} z\right)-c^{2}(y-2 c z) R^{2}=\dot{\theta}_{1}\left(-R^{1}\right)\left(R^{2}-R^{1}\right)-\theta_{1}\left(-R^{1}\right)-\theta_{2}\left(-R^{2}\right), \\
& \left(x-c^{2} z\right)-c^{2}(y-2 c z) R^{1}=\dot{\theta}_{2}\left(-R^{2}\right)\left(R^{1}-R^{2}\right)-\theta_{1}\left(-R^{1}\right)-\theta_{2}\left(-R^{2}\right) .
\end{aligned}
$$

In particular, it implies

$$
\boldsymbol{R}=\boldsymbol{R}\left(x-c^{2} z, y-2 c z\right),
$$

so that $\boldsymbol{R}$ is constant on the straight lines

$$
\vec{x}:=(x, y, z)=\left(x_{0}, y_{0}, 0\right)+\left(c^{2}+2 c, 1\right) s .
$$

We notice that starting from these solutions we may generate solutions

$$
u(x, y, z):=\int^{y} b_{0}(\boldsymbol{R}) \mathrm{d} y+\int^{z} b_{1}(\boldsymbol{R}) \mathrm{d} z,
$$

of the nonlinear equation

$$
u_{y y}=u_{u} u_{z x}-u_{y x} u_{z} .
$$




\section{Acknowledgements}

The authors thank Professor Eugeni Ferapontov for useful discussions. A.B. Shabat was supported by a grant of the Ministerio de Cultura y De-

porte of Spain. L. Martinez Alonso was supported by the DGCYT project BFM2002-01607.

\section{References}

[1] L. Martinez Alonso and A. B. Shabat, Phys.Lett. A 300, 58 (2002).

[2] L. Martinez Alonso and A. B. Shabat, J. Non. Math. Phys. 10 No 2, 1 (2003).

[3] L. Martinez Alonso and A. B. Shabat, "On the prolongation of a hierarchy of hydrodynamic chains" to appear in New Trends in Integrability and Partial Solvability proceedings NATO ARW.978791, NATO Science Series, Kluwer Academic Publishers.

[4] A. Shabat, Theor. and Math. Phys. 136 No 2, 1066 (2003).

[5] V. G. Mikhalev, Funct. Anal. Appl. 26 No 2, 140 (1992).

[6] M. Jaulent and I. Miodek, Lett. Math. Phys. 1, 243 (1976); Lett. Nuovo Cimento 20, 655 (1977).

[7] L. Martínez Alonso, J. Math. Phys. 21, 2342 (1980).

[8] M. Antonowicz and A. P. Fordy, Physica 28 D, 345 (1987).

[9] A. N. W. Hone, Phys. Lett. A 249, 46 (1998).

[10] R. Camassa and D. Holm, Phys. Rev. Letters 71, 1661 (1993).

[11] J. Gibbons and Y. Kodama. Phys. Lett. A 135, 167 (1989).

[12] J. Gibbons and S. P. Tsarev. Phys. Lett. A 211 , 19 (1996).

[13] J. Gibbons and S. P. Tsarev. Phys. Lett. A 258 , 263 (1999). 
[14] M. Mañas, L. Martinez Alonso and E. Medina. J. Phys. A: Math. Gen. 35, 401 (2002)

[15] E. V. Ferapontov and K. R. Khusnutdinova. On integrability of $(2+1)$ dimensional quasilinear systems nlin.SI/0305044, (2003).

[16] M. V. Pavlov. Integrable hydrodynamic chains nlin.SI/0301010, (2003).

[17] S. P. Tsarev. Izvestija AN USSR Math. 54, N5, 1048 (1990). 